\title{
RST ROBUST WATERMARKING SCHEMA BASED ON IMAGE NORMALIZATION AND DCT DECOMPOSITION
}

\author{
Faraoun Kamel Mohamed ${ }^{1}$, Rabhi Abbes ${ }^{2}$ \\ ${ }^{1}$ Evolutionary Engineering and Distributed Information Systems Laboratory, EEDIS \\ UDL University SBA, 22000 Algeria. Email: Fr_kamel@univ-dba.dz \\ ${ }^{2}$ Laboratoire des mathématiques, \\ UDL University SBA, 22000 Algeria. Email: rabhi_abbes@univ-sba.dz
}

\begin{abstract}
Geometric distortions are generally simple and effective attack to many existing watermarking methods which can make detection of the embedded watermark difficult or even impossible. A robust watermarking system must be able to encounter such attacks based on rotation, scaling and translation operators (RST attacks). In this paper, we describe a new robust watermarking schema based on logo embedding in the DCT transformed domain using image normalization techniques. In contrast to existing approaches, the watermark is not embedded directly in the normalized image. The image normalization is just used for calculating the affine transform parameters so that the watermark embedding and detection is performed in the original coordinates system. The performed experiments show that the proposed algorithm is robust against various types of attacks such as low-pass, median, Gaussian noise, aspect ratio change, rotation, scaling, JPEG compression, and the combination of these attacks.
\end{abstract}

\section{Keywords: Watermarking, Images normalization, DCT transformation}

\section{$1.0 \quad$ INTRODUCTION}

The tremendous growth in computer networks and the World Wide Web, coupled with the exponential increase of computer performance, has facilitated the distribution of multimedia data such as images. Publishers, artists, and photographers, however, may be unwilling to distribute pictures over the Internet due to a lack of security as images can be easily duplicated and distributed without the owner's consent. Digital watermarks have been proposed as a way to tackle this tough issue. This digital signature could discourage copyright violation, and may help determine the authenticity and ownership of an image.

In general, a digital watermark is a code that is embedded inside an image. It acts as a digital signature, giving the image a sense of ownership or authenticity. Ideal properties of a digital watermark have been stated in the literature $[7,4,8,13]$. These properties include: perceptual invisibility to prevent obstruction of the original image, statistical invisibility, fairly simple extraction, robustness to filtering, additive noise, compression, and other forms of image manipulation as well as the ability to determine the owner of the original image.

Many of the current techniques for embedding marks in digital images have been inspired by methods of image coding and compression. Information has been embedded using the Discrete Cosine Transform (DCT) [1, 2], Discrete Fourier Transform magnitude and phase [3], wavelets [6], Linear Predictive Coding [4] and Fractals [5] . The key to making watermark robust has been the recognition that in order for a watermark to be robust it must be embedded in the perceptually significant components of the image [7]. The term "perceptually significant" is somewhat subjective but it suggests that a good watermark is one witch takes account of the behavior of human visual system

In order for a watermark to be useful, it must be robust against a variety of possible attacks. These include robustness against compression such as JPEG, scaling and aspect ratio changes, rotation, cropping, row and column removal, addition of noise, filtering, cryptographic and statistical attacks, as well as insertion of other watermarks. While many methods perform well against compression, they lack robustness to geometric transformations. Rotation and scaling attacks are considered more challenging than other attacks. Usually, the embedding of a strong watermark improves the delectability against the image compression and filtering attacks. However, different from these kinds of attacks, geometric manipulations are difficult to tackle. The main difficulty in geometric attacks is loss of synchronization in the watermark detector. Thus, the detection fails even though the watermark still exists in the watermarked image. Those are challenging attacks in that they do not introduce the quality degradation very much but make the detection process very complex and difficult. 
In this paper, we describe a robust logo embedding algorithm that is resistant to geometric attacks. The synchronization is recovered using an image normalization technique for the detection process. Instead of embedding the watermark in the normalized image directly, an idea of image normalization is used to calculate the affine transform parameters so that the watermark embedding and detection is performed in the original coordinates system. In addition, for a maximum watermark embedding with least perceptual degradation, a developed visual masking is used. The watermarking structure is based on a DCT transform method, and so the watermarking schema is semi-blind and an original image during the detection process is not needed. For robust watermark detection, an optimum threshold with a given false detection error probability is presented so that the threshold can be determined in advance regardless of the attacks that the watermarked image has undergone. It is useful to determine the optimal threshold in advance because the decoder complexity for calculating the threshold can be reduced.

The paper is organized as follows. In section 2, a related theory about the DCT block parent-child structure and image normalization is presented. Section 3 presents the existing related works to the RST invariants watermarking systems. In section 4, the proposed approach is described, which includes watermark insertion and detection mechanisms. Experimental results and discussions are given in section 5, and the conclusions drawn are presented in section 6 .

\subsection{RELATED THEORY}

\subsection{DCT image transformation}

Digital image watermarking technology is closely related to image coding technology. Transform coding is now the de-facto standard in image and video coding, while the Discrete Cosine Transform (JPEG, MPEG-1, 2,H.261, H.263) and the Discrete Wavelet Transform (JPEG2000) are mostly used. Given an image A of size Mx N, the DCT of the image is defined as [8]:

$$
B\left(k_{1}, k_{2}\right)=\sum_{i=0}^{M-1} \sum_{j=0}^{N-1} 4 \cdot A(i, j) \cdot \cos \left[\frac{\pi \cdot k_{1}}{2 \cdot M}(2 i+1)\right] \cdot \cos \left[\frac{\pi \cdot k_{2}}{2 \cdot M}(2 j+1)\right]
$$

$\mathrm{B}\left(\mathrm{k}_{1}, \mathrm{k}_{2}\right)$ is the DCT coefficient of the image in row $\mathrm{k}_{1}$ and column $\mathrm{k}_{2}$. Larger DCT coefficients are usually located at low frequencies (upper left corner), while coefficients at high frequencies are very small. That is, DCT puts most of an image's energy at low frequencies.

DWT separates an image into several sub-images corresponding to horizontal, vertical and diagonal details at each resolution level and a coarsest resolution level. Like DCT, most energy of an image is put in the coarsest sub-image.

\subsection{Image Normalization}

The typical geometrical attacks include rotation, scaling and translation of the image. These kinds of attacks can be represented by affine transform. The affine transform with scaling parameters (a,b), rotation angle $\varphi$ and translational parameters ( $\mathrm{Tx}, \mathrm{Ty})$ can be defined as:

$$
\left[\begin{array}{l}
x_{a} \\
y_{a}
\end{array}\right]=\left[\begin{array}{ll}
a & 0 \\
0 & b
\end{array}\right]\left[\begin{array}{cc}
\cos \varphi & -\sin \varphi \\
\sin \varphi & \cos \varphi
\end{array}\right]\left[\begin{array}{l}
x \\
y
\end{array}\right]+\left[\begin{array}{l}
T_{x} \\
T_{y}
\end{array}\right]
$$

where $(x, y)$ are the pixel coordinates of an input image and $\left(x_{a}, y_{a}\right)$ are the pixel coordinates of a transformed image. The affine transform parameters can be estimated using image moments. The image moment of order $p+q$ is defined in the two dimensional Cartesian coordinates as:

$$
m_{p q}=\sum_{y=0}^{N_{2}-1} \sum_{x=0}^{N_{1}-1} x^{p} y^{q} \cdot I(x, y)
$$

The centroid of the image can be calculated using the zeroth and the first moments: 


$$
\bar{x}=m_{10} / m_{00} \quad, \quad \bar{y}=m_{01} / m_{00}
$$

The translational effect in the image can be removed by setting the transform center as $(\bar{x}, \bar{y})$. The central moments of order $p+q$ is defined as:

$$
\mu_{p q}=\sum_{y=0}^{N_{2}-1} \sum_{x=0}^{N_{1}-1}(x-\bar{x})^{p}(y-\bar{y})^{q} \cdot I(x, y)
$$

Now, we can normalize the input image of size $1_{x} \times 1_{y}$ using the image moments. Let the width and the height of the normalized image be $\hat{l}_{x}=a l_{x}$ and $\hat{l}_{y}=b l_{y}$ so that the aspect ratio of the normalized image should be one.

$$
\hat{y}=b \cdot l_{y} / a \cdot l_{x}=1
$$

If the aspect ratio of the input image is $y=l_{y} / l_{x}$, we can get by replacing the relation into equation:

$$
a=b \cdot y
$$

Let $I(x / a, y / b)$ be the normalized image of the input image $I(x, y)$. Then, the zeroth moment of the normalized image can be obtained by changing the variables in equation (2):

$$
\tilde{m}_{o o}=a \cdot b \cdot m_{00}
$$

By solving the simultaneous equations of (5) and (6), finally we can calculate the scaling parameters.

$$
a=\sqrt{y \cdot \hat{m}_{00} / m_{00}} \quad, \quad b=\sqrt{\hat{m}_{00} / y \cdot m_{00}}
$$

The image normalization against rotation can be performed using tensor theory defined in [9]. The rotation angle $\varphi$ for image normalization can be calculated using the following equations:

$$
\begin{aligned}
& t^{1}=\mu_{21}+\mu_{30} \text { and } t^{2}=\mu_{03}+\mu_{21} \\
& \varphi=\tan ^{-1}\left(-t^{1} / t^{2}\right)
\end{aligned}
$$

Equation (8) has two possible solutions, thus, we choose $\varphi$ such that $-t^{1} \sin \varphi+t^{2} \cos \varphi>0$ to ensure a unique solution.

We can transform any input image to a normalized form by identifying the transform parameters, $(a, b), \varphi$ and $(\bar{x}, \bar{y})$. If two different images are an affine transform pair, the normalized form of these images will be the same.

\subsection{RELATED WORKS}

Recently, major of the researches concerning watermarking robustness improvement are interested to RST invariants metodes. O'Ruanaidh et al. [10] have outlined the theory of integral transform invariants and showed that this can be used to produce watermarks that are resistant to rotation, scaling, and translation. In their approach, the discrete Fourier transform (DFT) of an image is computed and then the Fourier-Mellin transform is performed on the magnitude, the watermark is embedded in the magnitude of the resulting transform. The watermarked image is reconstructed by performing the inverse transforms (an inverse DFT and an inverse Fourier-Mellin transform) after considering the original phase [11][10]. Fourier-Mellin transform is a log-polar mapping (LPM) followed by a Fourier transform, while an inverse Fourier-Mellin transform is an inverse log-polar mapping (ILPM) followed by an inverse Fourier transform. In the scheme, the embedded watermark may be extracted by transforming the watermarked image into RST invariant domain. However, O'Ruanaidh et al. [10] noted very severe implementation difficulties which might have hampered further work in this area. Pereira et al. [13] proposed to embed two 
watermarks, a template and a spread spectrum message containing the information or payload. The template contains no information itself, but is used to detect transformations undergone by the image. One problem with this solution is that this approach is likely to reduce the image quality, because it requires the insertion of a registration watermark in addition to the data-carrying watermark, Lin et al. [12] proposed a method that develops a watermark invariant to geometric distortions, and that eliminates the need to identify and invert them. The watermark is embedded into a translation and scaling invariant one-dimensional signal obtained by taking the Fourier transform of the image, re-sampling the Fourier magnitudes into log-polar coordinates, and then summing a function of those magnitudes along the log-radius axis.

In $[14,15], Z$. Dong has proposed to embed watermark in the log-polar mappings of Fourier magnitude spectrum of original image, and use the phase correlation between the LPM of the original image and the LPM of the watermarked image to calculate the displacement of watermark positions in LPM domain. The scheme preserves the image quality by avoiding computing inverse log-polar mapping (ILPM).

In [16], a watermarking scheme is implemented by improving image normalization based watermarking (INW). Image normalization is based on the moments of the image, Invariant Centroid (IC) is proposed and the only central region(R), which has less cropping possibility by RST, is used for normalization.

J. Xuan and H.Zhang [17] proposed a rotation, scaling and translation (RST) resilient watermarking method through embedding watermark in RST invariant derived from Radon transform and Fourier transform. Based on the translation and rotation properties of Radon transform and the translation invariant property of Fourier magnitude, the RST invariant is obtained.

\subsection{PROPOSED APPROACH}

In the following, details of the proposed watermarking schema are explained. The two main phases of any watermarking system are embedding of the watermark and its extraction or detection process. In the proposed method, the embedded watermark is a Logo binary image of size $64 x 64$, where the host images used for benchmarking are gray scale $512 \times 512$ bitmaps.

\subsection{Watermark embedding}

The block diagram for watermarks embedding is shown in Fig. 1. The original image is transformed to $8 \times 8$ block DCT domain. Then, the robust and the fragile watermarks are embedded in the DCT block. For robust logo embedding, the perceptual weighting for each block is calculated using spatio-frequency localization property of the $8 \times 8$ DCT block. Two randomly selected groups of coefficients in each block are modified to embed one bit of information. The fragile watermark is embedded into the high frequency DCT coefficients which are vulnerable to the image modifications. To cope with geometric manipulations, the zeroth moment $\left(m_{00}\right)$ of the watermarked image and rotation angle $(\varphi)$ between the watermarked image and its normalized image are calculated. For the security of the embedding procedure, the binary $\operatorname{logo} S=\left\{s_{0}, s_{1}, \ldots, s_{M-1}\right\}$ is modulated with the pseudorandom bit sequence $R=\left\{r_{0}, r_{1}, \ldots, r_{M-1}\right\}$ to generate the modulated watermark sequence $P=\left\{p_{0}, p_{1}, \ldots, p_{M-1}\right\}$, where $s_{i}, r_{i}, p_{i} \in\{0,1\}$. The modulation is based on the bit-wise logical XOR operation: $p_{\mathrm{i}}=s_{i} \oplus r_{i}$

The embedding is based on the two set operation, i.e., we embed one bit of watermark according to the sign of difference between two groups of randomly selected coefficients in the $8 \times 8$ block. Let $G_{X}$ and $W_{X}$ be coefficients and their corresponding weighting values of a group $\mathrm{X}$ respectively. Then, the two groups $G_{A}$ and $G_{B}$ can be represented as:

$$
\begin{array}{ll}
G_{A}=\left\{a_{1}, a_{2}, \ldots, a_{N-1}\right\} & W_{A}=\left\{u_{1}, u_{2}, \ldots, u_{N-1}\right\} \\
G_{B}=\left\{b_{1}, b_{2}, \ldots, b_{N-1}\right\} & W_{B}=\left\{v_{1}, v_{2}, \ldots, v_{N-1}\right\}
\end{array}
$$

where $\mathrm{N}$ is the number of elements in each group. 


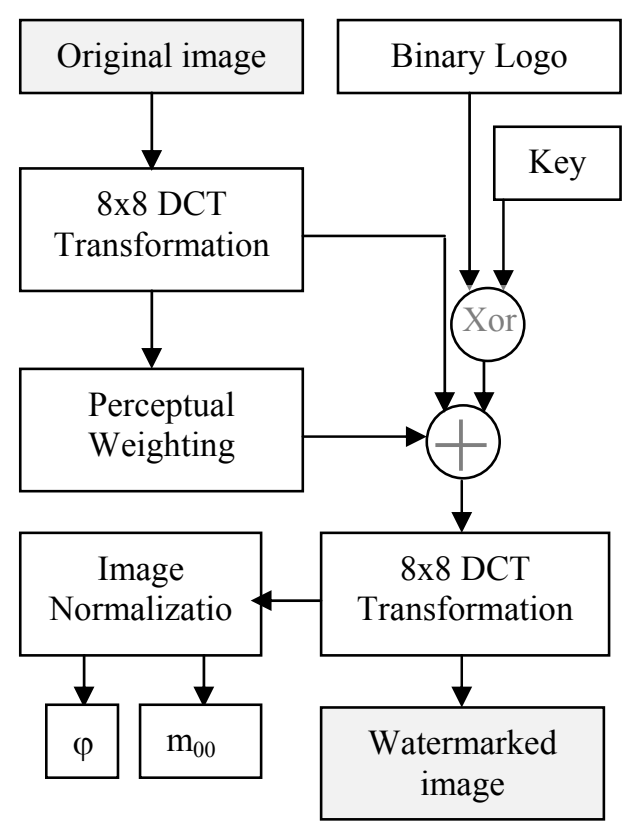

Fig. 1: General block diagram for the watermark (logo) embedding process

When we embed $p_{j}=^{\text {' }} 1$ ' into the block $j$, we increase the absolute values of the coefficients in $G_{A}$ by an amount of corresponding scaled (with $\alpha$ ) weights in $W_{A}$ and decrease the absolute values of the coefficients in $G_{B}$ by an amount of corresponding scaled weights in $G_{B}$ until $A S D_{j}\left(G_{A}, G_{B}\right)$ (Absolute Sum Difference between $G_{A}$ and $G_{B}$ ) is greater than zero or predefined iteration is reached. On the other hand, when we embed " 0 " we decrease the absolute values of the coefficients in $G_{A}$ by an amount of corresponding scaled weights in $W_{A}$ and increase the absolute values of the coefficients in $G_{B}$ by an amount of corresponding scaled weights in $W_{B}$ until $A S D_{j}\left(G_{A}, G_{B}\right)$ is smaller than zero, or predefined iteration is reached. The $A S D_{j}\left(G_{A}, G_{B}\right)$ in the block $j$ is defined as:

$$
A S D_{j}\left(G_{A}, G_{B}\right)=\sum_{i=0}^{N-1}\left|a_{i}\right|-\sum_{i=0}^{N-1}\left|b_{i}\right|
$$

pseudo-code for the embedding procedure is depicted in Fig. 2. In the code, the functions $\operatorname{sgn}(x)$ and $\operatorname{step}(x)$ are well-known signum and unit step functions respectively.

$$
\begin{gathered}
\operatorname{sgn}(x)=\left\{\begin{array}{cc}
1 & \text { when } x>0 \\
0 & \text { when } x=0 \\
-1 & \text { when } x<0
\end{array}\right. \\
\text { step }(x)=\left\{\begin{array}{l}
1 \text { when } x \geq 0 \\
0 \text { when } x<0
\end{array}\right.
\end{gathered}
$$

Three transform parameters have to be identified to invert the geometric manipulations. The translation can be compensated by calculating the image centroid $(\bar{x}, \bar{y})$. The scale and rotation can be compensated by using equation (7) and (8). Thus, we need $m_{00}$ and $\varphi$ of the watermarked image in watermark detection process. 


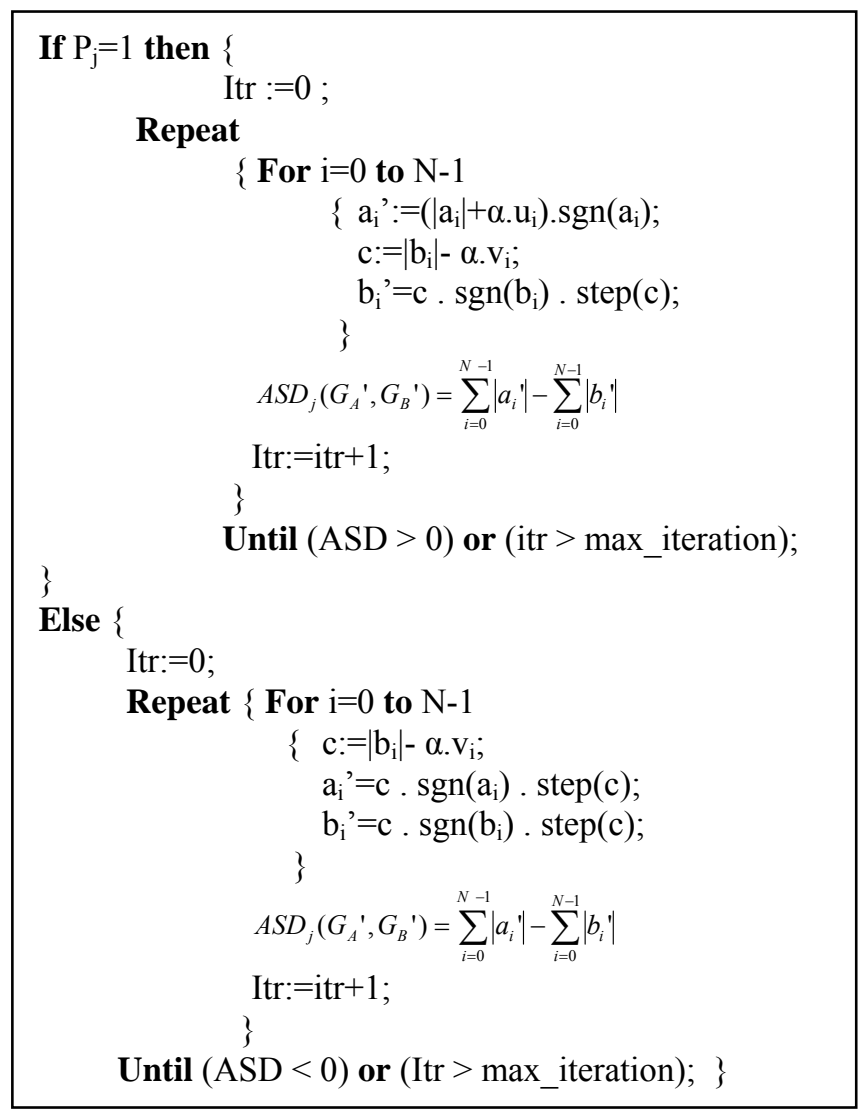

Fig. 2: The used bit embedding algorithm

\subsection{Watermark extraction process}

The logo extraction is a reverse process of embedding. The image that is watermarked and possibly corrupted by attacks is normalized, and then scale parameters and rotation angles are calculated using equation (7) and (8) respectively. After inverting the geometric transform, the image is transformed into $8 \times 8 \mathrm{DCT}$ domain. Then, the block by block bit extraction is performed. For each block $j, A_{S_{j}}\left(G_{A}, G_{B}\right)$ is calculated. If $A_{S}\left(G_{A}, G_{B}\right) \geq 0$ then, we can decode $p_{j}$ as ' 1 ' else decode $p_{j}$ as ' 0 '. Fig. 3 illustrates the general schema of the logo detection process.

Apart from extracted logo, the matching score using traditional normalized cross correlation between the original watermark and the decoded watermark [18] can be calculated. The score is computed as follows:

$$
S=\frac{\sum_{i=0}^{M-1}\left(2 p_{i}-1\right)\left(2 p_{i}{ }^{\prime}-1\right)}{\sqrt{\sum_{i=0}^{M-1}\left(2 p_{i}-1\right)^{2} \sum_{i=0}^{M-1}\left(2 p_{i}{ }^{\prime}-1\right)^{2}}}=\frac{1}{M} \sum_{i=0}^{M-1}\left(2 p_{i}-1\right)\left(2 p_{i}{ }^{\prime}-1\right)
$$

where $p_{i}$ and $p_{i}^{\prime}$ are the original and the extracted watermarks respectively. $M$ is the length of watermark. If the score $s$ is higher than a certain threshold $T_{s}$, we can say that the watermark is present in the image. However, how much higher response is required for the watermark to exist? To solve this problem we present a method to calculate an optimal threshold for declaring the existence of the watermark with a given probability of false detection. To do this, it is assumed that $s$ is normally distributed with a mean and a variance of $\mu_{s}$ and $\sigma_{s}^{2}$ respectively. The detection of watermark is modeled as a hypothesis testing problem. We have two cases for the null hypothesis (H0: an image $I^{\prime}(x, y)$ is not watermarked with $\left.X\right)$. The first case is that the image, $I^{\prime}(x, y)$ is not watermarked. The second case is that the image $I^{\prime}(x, y)$ is watermarked with $Y$ other than $X$. In our problem, the both cases are similar because regardless of other watermark embedding, the distributions of $p_{i}$ are same, i.e. in any case $p_{i}$ is uniformly distributed with equal probabilities of $\operatorname{Pr}\left(p_{j}=0\right)=0.5$ and $\operatorname{Pr}\left(p_{j}=1\right)=0.5$. 


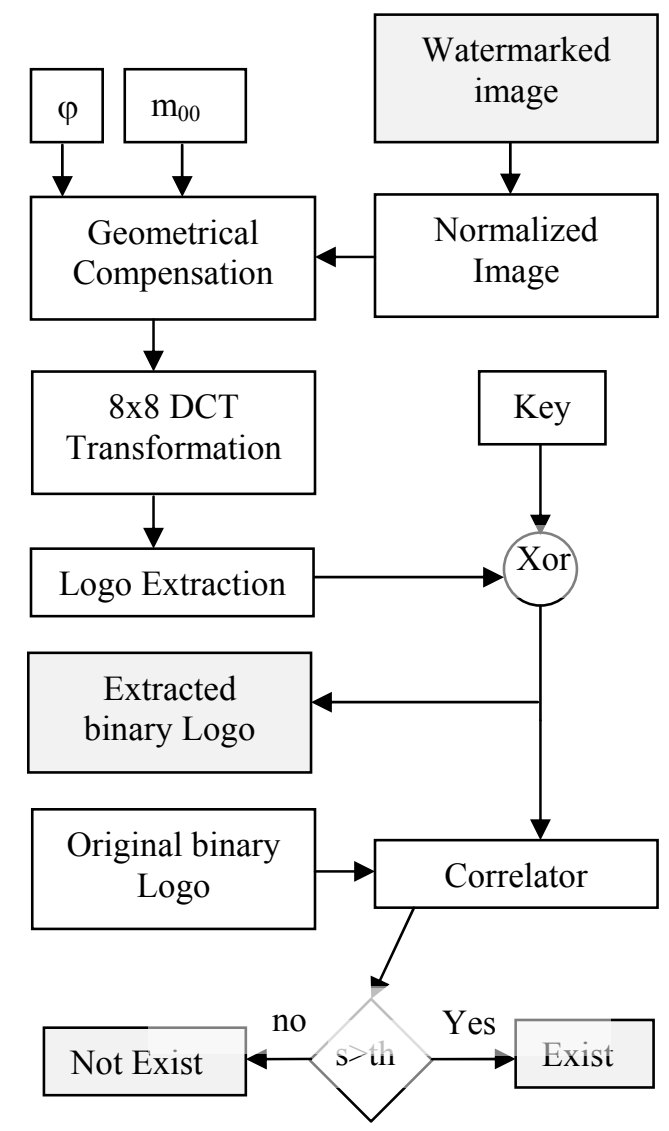

Fig. 3: Block diagram for watermark detection process

With the assumption that $\mathrm{s}$ is normally distributed, we can calculate the false detection probability as:

$$
P_{f}=\frac{1}{2} \operatorname{erfc}\left(T_{s}-\mu_{s} / \sqrt{2} \sigma_{s}\right)
$$

Using $\mu_{s}=0$ and $\sigma_{s}^{2}=1 / \mathrm{M}$, and threshold with $P_{f} \leq 10^{-10}$ can be calculated using equation (11), the following is obtained:

$$
T_{s}=4.5 \sqrt{2 . \sigma_{s}^{2}}
$$

The final threshold can be calculated by replacing the $\sigma_{s}^{2}=1 / \mathrm{M}$ into equation (13):

$$
T_{s}=4.5 \sqrt{2 / M}
$$

The threshold only depends on the length of the embedded bits. The result has a meaning that we can use the consistent threshold regardless of the original image and embedded watermark strength in the detection process. Fig. 4 and Fig. 5 show the experimental distribution and theoretical pdf (with $\mu_{s}=0$ and $\sigma_{s}^{2}=1 / \mathrm{M}$ ) of the detector output $s$. 


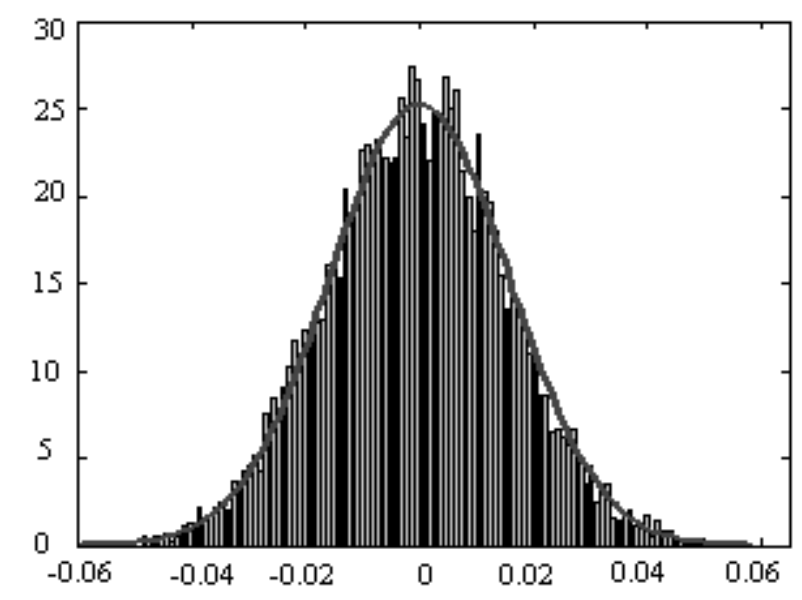

Fig. 4: Distribution of the detector $10^{4}$ output for unwatermarked image and its theoretical pdf

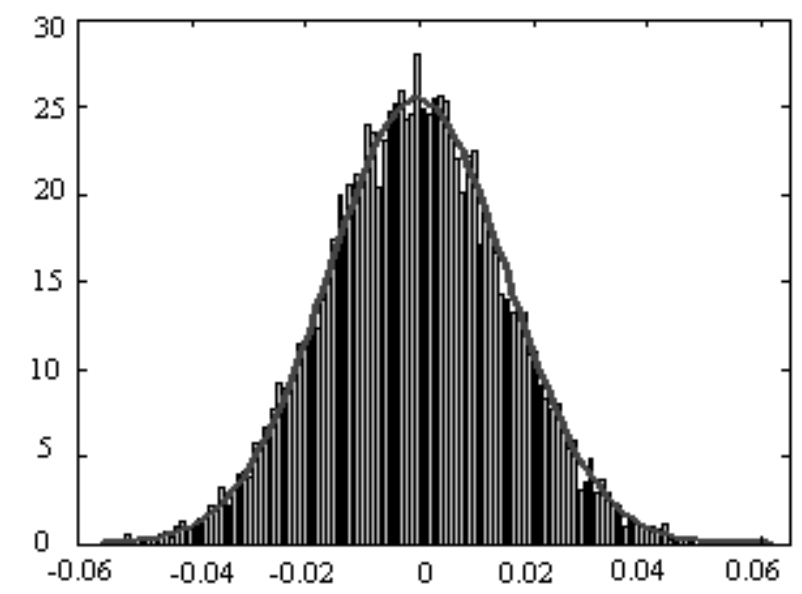

Fig. 5: Distribution of the detector $10^{4}$ output for the second case and its theoretical pdf

\subsection{RESULTS AND DISCUSSION}

The proposed algorithm was tested on the popular $512 \times 512$ Lena image as shown in Fig. 6(a). The image is watermarked with the logo shown in Fig. 8 (a). The size of logo is $64 \times 64$ so that one bit of information can be embedded into $8 \times 8$ block. The watermarked version of Fig. 6(a) is shown in Fig. 6(b). The PSNR (Peak Signal to Noise Ratio) between these two images was $42.0885 \mathrm{~dB}$ with embedding parameter $\alpha=2.5$. As can be seen from Fig. 6(a) and Fig. 6(b), the original and the watermarked image is perceptually indistinguishable. It means that the watermark is effectively masked. 


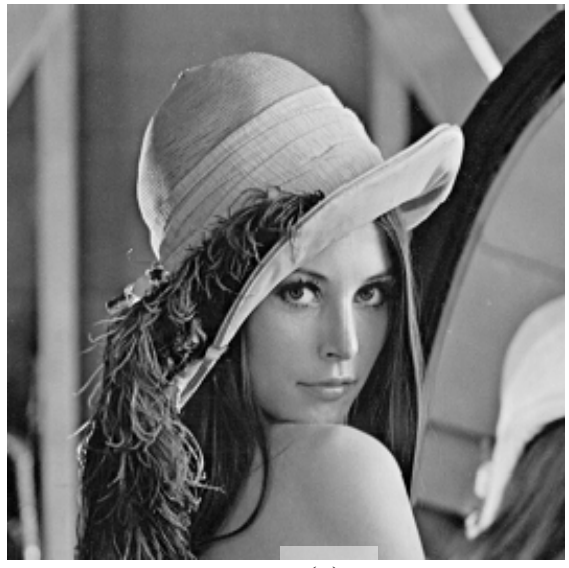

(a)

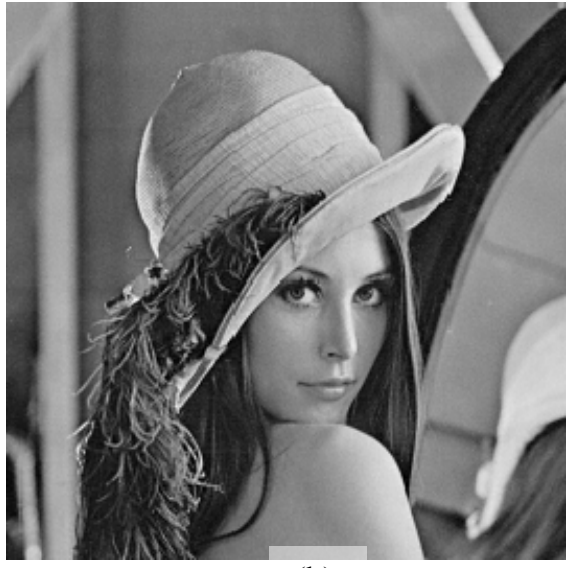

(b)

Fig. 6: (a) Original Lena image and (b) watermarked image

Fig. 7(a) shows the absolute difference between the original and the watermarked image. The difference was scaled up for the illustrative purpose. The figure indicates that the watermark is mainly embedded into the highly active region of the image, which is less sensitive to human eyes. Several signal processing attacks as well as geometric attacks were simulated to demonstrate the robustness of the algorithm. Fig. 7(b) shows the image attacked with $30^{\circ}$ rotation and 0.8 scaling.

The extracted logos from lowpass filtering, median filtering, and Gaussian noise attacks with $30^{\circ}$ rotation and 1.5 times scaling are shown in Fig. 8(c), Fig. 8(d) and Fig. 8(e) respectively. The extracted logos from aspect ratio change of 0.5 and 2 are shown in Fig. 8(f) and Fig. 8(g) respectively. Fig. 7(b) shows the image attacked with $30^{\circ}$ rotation and 0.8 scaling. Fig. 8(h) shows extracted logos from Fig. 7(b). Fig. 9 shows the corresponding matching score for extracted logos in Fig. 8 with 1000 different keys. The horizontal line in the figures indicates the threshold with false detection probability of $\mathrm{P}_{\mathrm{f}}<10^{-10}$.

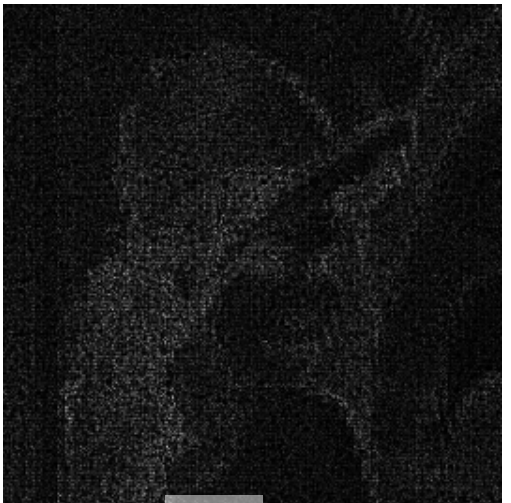

(a)

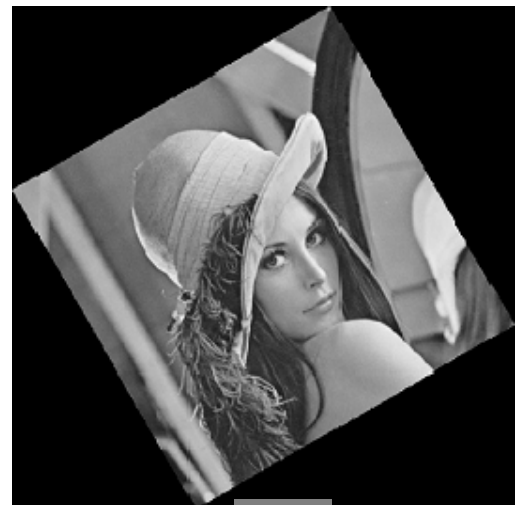

(b)

Fig. 7: (a) Absolute difference between 6(a) and 6(b), (b) rotated $\left(30^{\circ}\right)$ and scaled $(0.8)$ version of $6(\mathrm{~b})$

We can see that even if the attack is a combination of signal processing and geometrical transform, the detector output is reliable. Fig. 10 shows that our scheme can resist rotation attacks of any angle, by illustrating the detector response intensity against various possible attacks. The detector output for the scaling with varying magnification is shown in Fig. 11. As illustrated, the response has a local maximum at the integer multiples of magnifications. This is due to the interpolation in calculation of the magnifications. Fig. 12 and Fig. 13 show the detector response for rotation attacks with fixed magnification and scaling attacks with fixed rotation angle respectively. The detector is reliable for any combination of rotation and scaling. 


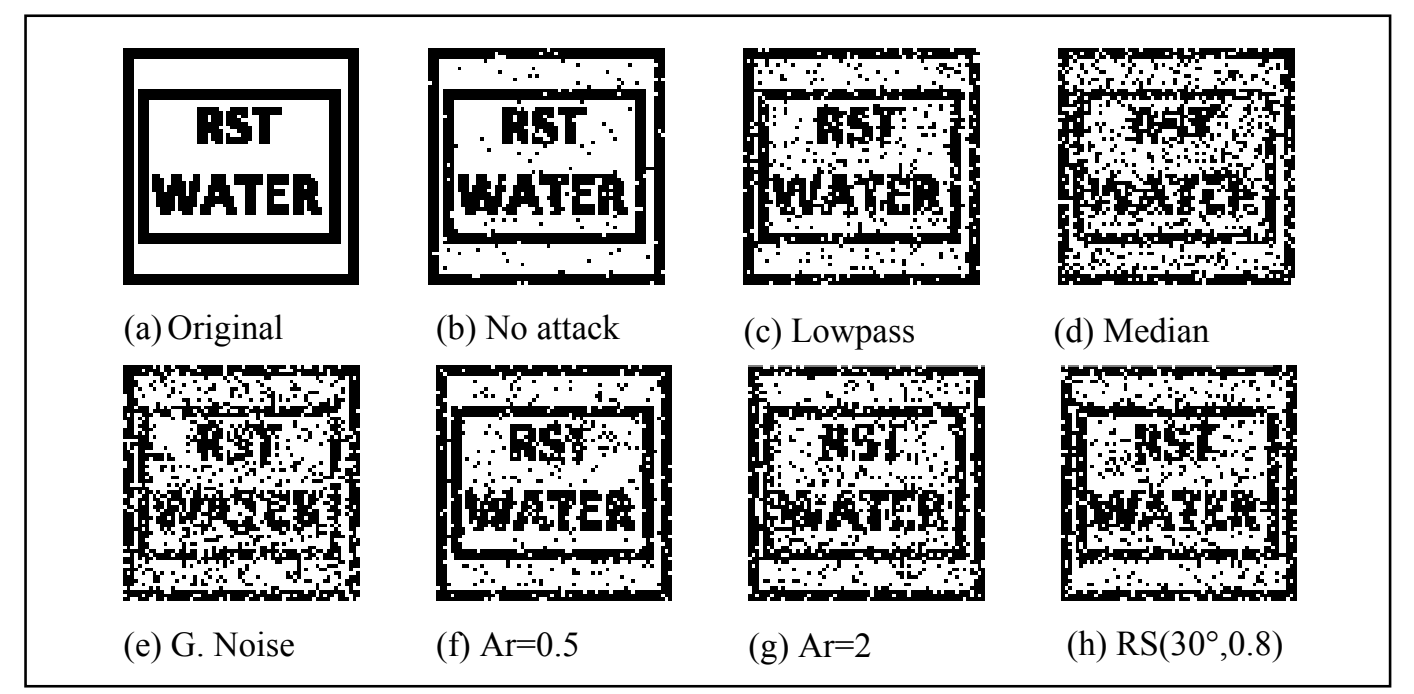

Fig. 8: Extracted logos from attacked images
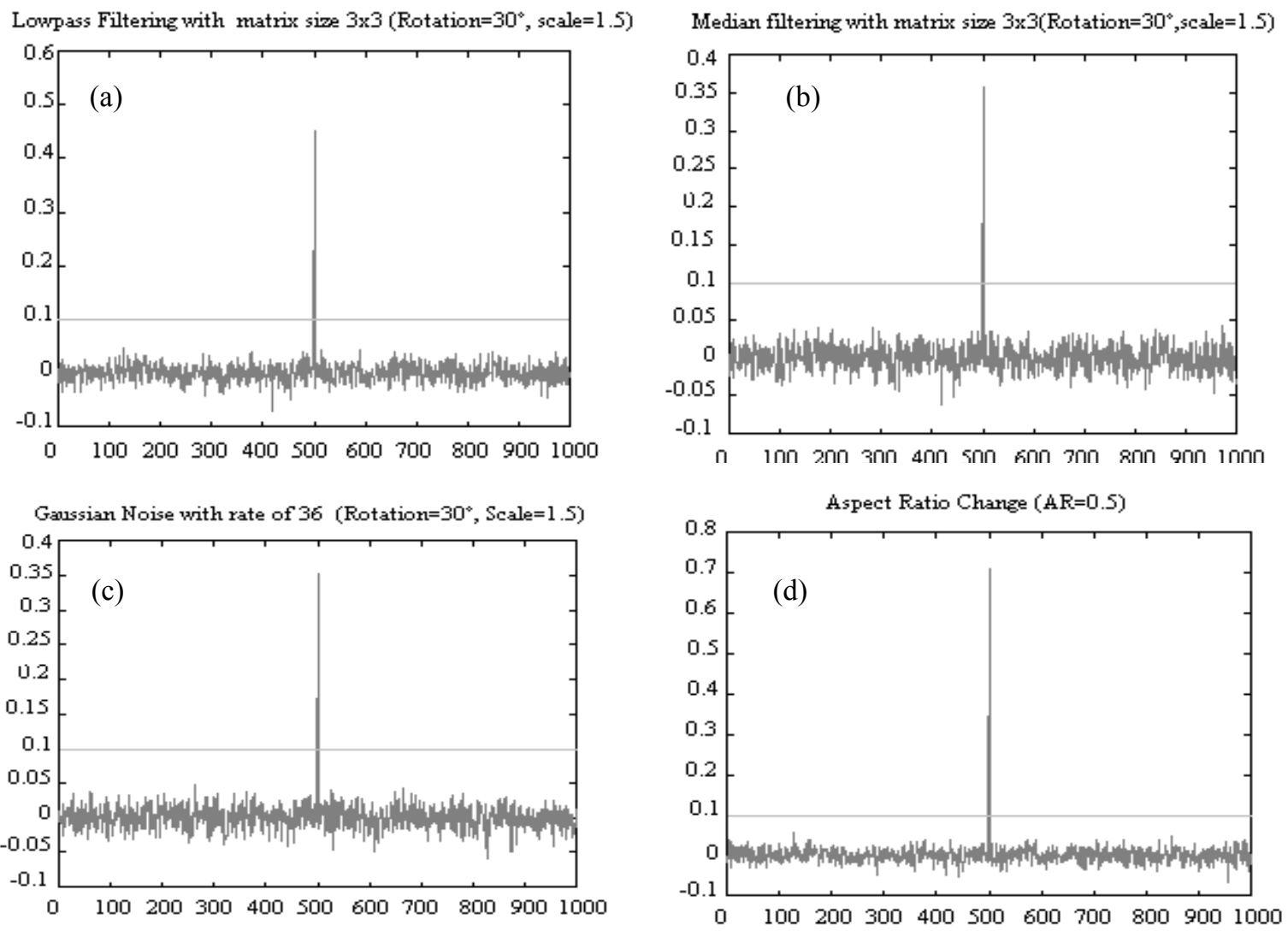

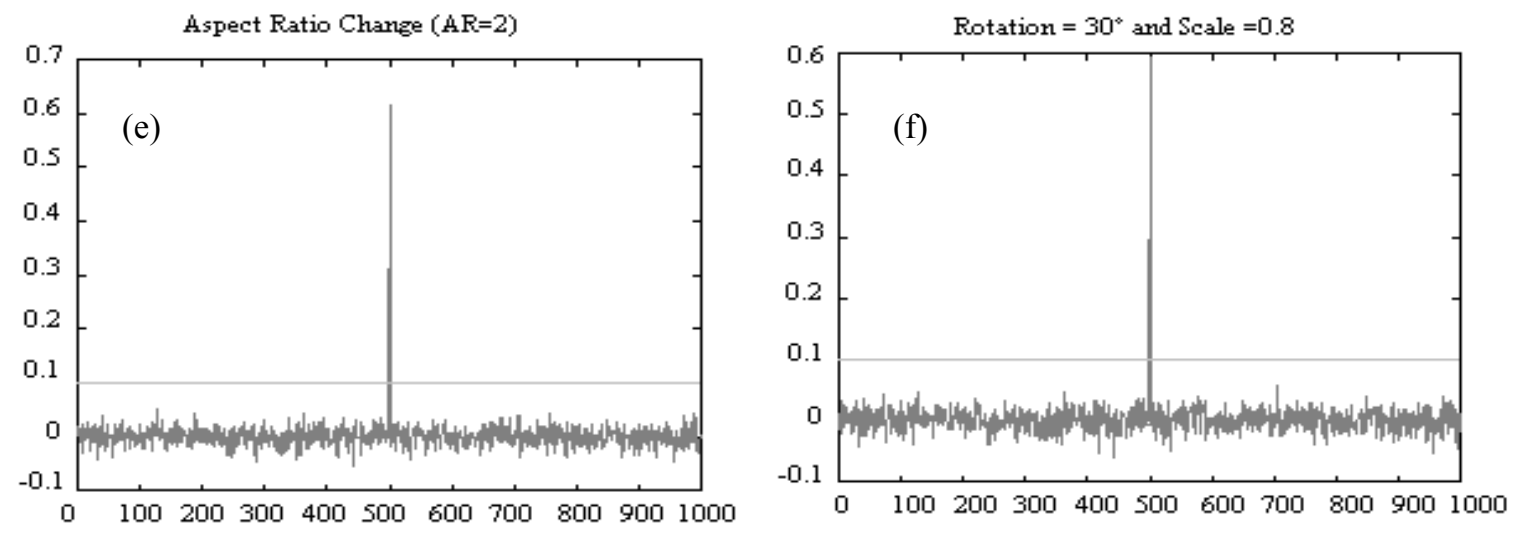

Fig. 9: Detector response for various attacks (a) Lowpass tilter, (b) Median Filter, (c) G. Noise, (d) As. Ratio 0.5, (e) As. Ratio 2, (f) Rotation $30^{\circ}$ and scale 0.8

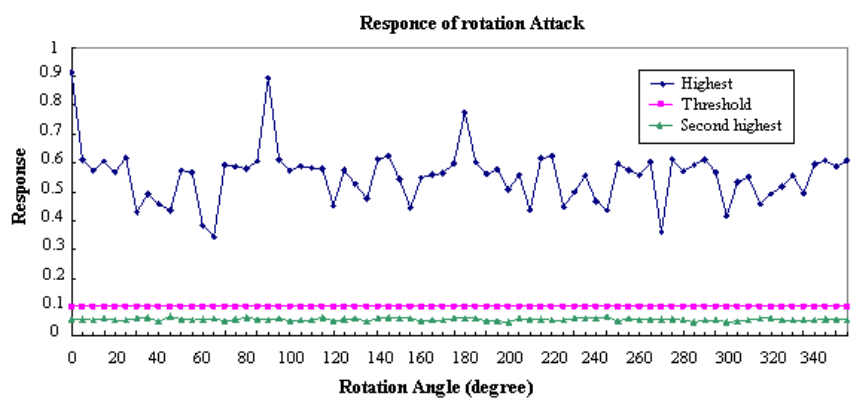

Fig. 10: Detector response for the rotation with varying angles

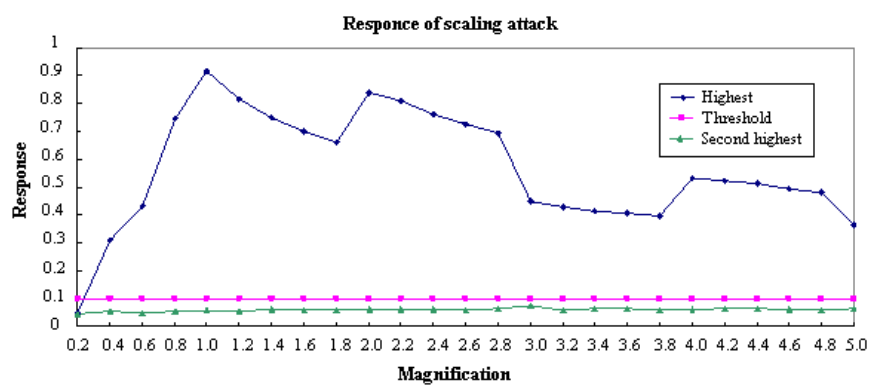

Fig. 11: Detector response for the scaling with varying magnification

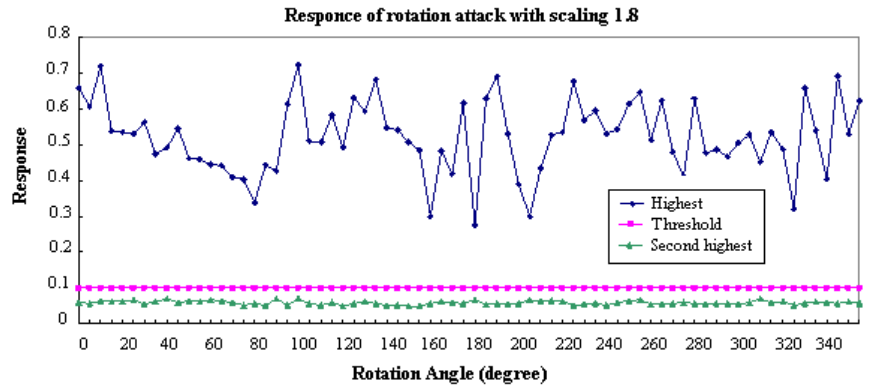

Fig. 12: Detector response for the rotation with scaling of 1.8 and varying angles 


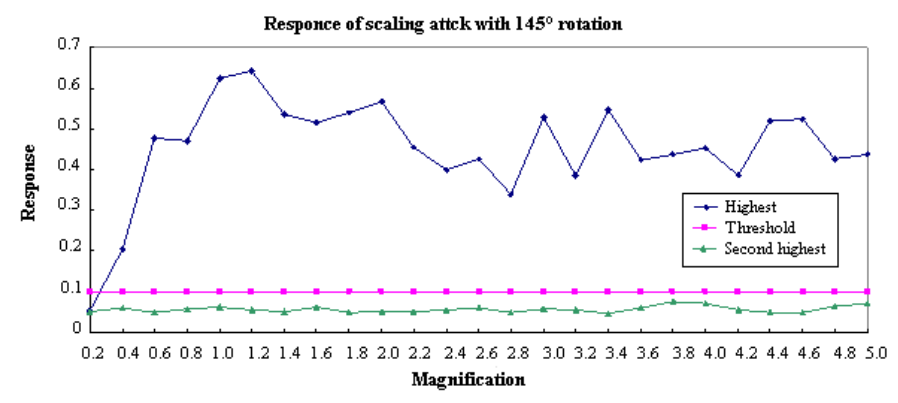

Fig. 13: Detector response for the scaling with $145^{\circ}$ rotation and varying magnification

The robustness for image compression is very important in image watermarking because almost all images are distributed in coded format. The detector response for JPEG compression with fixed rotation and scaling is shown in Fig. 14, where compression ratios are varying. Even if the image undergoes combination of geometric manipulation and compression, the detector shows robust performance until the compression ration reaches $3.1 \%$ of the original size.

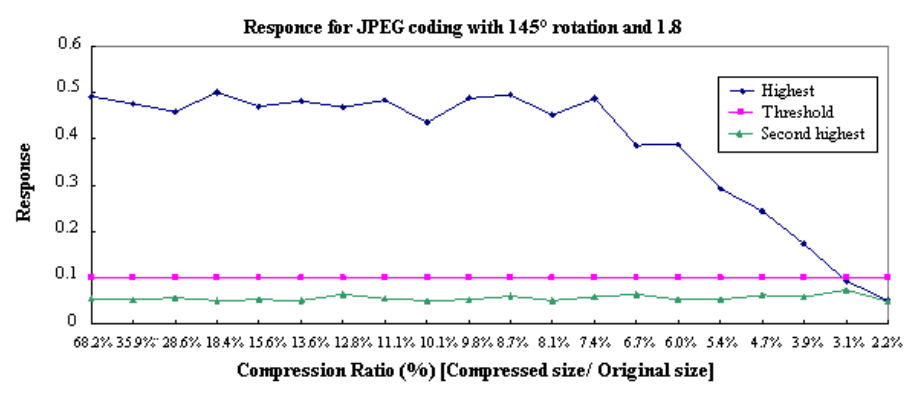

Fig. 14: Detector response for the JPEG coding with $145^{\circ}$ rotation, scaling of 1.8 and varying compression ratios

\subsection{CONCLUSION}

In this paper, we described a robust watermark embedding scheme using image normalization. To avoid the quality degradation caused by interpolation in image normalization, we do not embed the watermark in the normalized image. Instead, we use the idea of image normalization just for calculating the affine transform parameters so that the watermark embedding and detection can be performed in the original coordinates system. The robustness of the algorithm came from the utilization of the HVS property. The optimum threshold with a given false detection probability was presented so that we can determine the threshold in advance regardless of the attacks that watermarked image has undergone. We tested algorithm for various types of attacks such as lowpass, median, Gaussian noise, aspect ratio change, rotation, scaling, JPEG compression, and the combination of these attacks. Simulation results showed that the proposed algorithm is robust and reliable against various attacks and geometric transformation.

\section{REFERENCES}

[1] A. Bors, I. Pitas. "Image Watermarking using DCT Domain Constraints", IEEE International Conference on Image Processing (ICIP'96), Lausanne, Switzerland, Vol. III, 16-19 September 1996, pp. 231-234,.

[2] M. Barni, F. Bartolini, V. Cappellini, A. Piva. "A DCT Domain System for Robust Image Watermarking", Signal Processing, Vol. 66, 1998, pp. 357-372. 
[3] J. J. K. O Ruanaidh, W. J. Dowling and F. M. Boland. "Phase watermarking of digital images" in Proceedings of the IEEE International conference of Image Processing ICIP-96, Lausanne, Switzerland, September 1996, pp. 239-242.

[4] K. Matsui and K. Tanaka. "Video-Steganography: How to secretly embed a signature in a picture" in $I M A$ Intellectual Property Project Proceedings, January 1994, pp. 187-206.

[5] P. Devern and M. Scott. "Fractal based images steganography" in Proceedings of the First International Workshop in Information Hiding, Ross Anderson (Ed.), Lecture notes in computer sciences, Cambridge, UK, May/June 1996, pp. 279-294.

[6] R. Eslami and H. Radha, "New Image Transforms Using Hybrid Wavelets and Directional Filter Banks: Analysis and Design," in Proceedings of IEEE International Conference on Image Processing, Vol. 1, 2005, pp. 733-736.

[7] A. Michael, M. Schmucker and D.Wolthusen, "Techniques and applications and Digital Watermarking and content protection", Brithich library Cataloguing in publication data, Artech House computer security series, ISBN 1-58053-111-3. 685 Canton Street, Norwood, MA 02062.

[8] Z. Xiong, K. Ramchandran, M. T. Orchard and Y Zhang, "A comparative study of DCT- and wavelet-based image coding”, IEEE Trans. on Circuits and Systems for Video Technology, Vol.9 No.5, August, 1999.

[9] S. C. Pei and C. N. Lin, "Image Normalization for Pattern Recognition”, Image and Vision Computing, Vol. 13, No. 10, December 1995, pp. 711-723.

[10] J. O'Ruanaidh and T. Pun, "Rotation, Scale, and Translation Invariant Digital Image Watermarking”, Signal Processing, Vol. 66 No. 3, 1998, pp. 303-317.

[11] M. Alghoniemy and A. Tewfik. "Progressive Quantized Projection Watermarking Scheme" in Proceedings of ACM Multimedia 99, Vol. 1, 1999, pp. 295-298.

[12] C. Lin, M. Wu, J. Bloom, I. Cox, M. Miller, and Y Lui, "Rotation, Scale, and Translation Resilient Watermarking for Images”, IEEE Transactions on Image Processing, Vol. 10, No. 5, 2001, pp. 767-782.

[13] S. Pereira and T. Pun, "Robust Template Matching for Affine Resistant Image Watermarks", IEEE Transactions on Image Processing, Vol. 9 No. 6, 2000, pp. 1123-1129.

[14] D. Zheng, J. Zhao, A. El Saddik, "RST invariant digital image watermarking based on log-polar mapping and phase correlation", IEEE Transactions on Circuits and Systems for Video Technology, Vol. 9 No.6, September 2003.

[15] D. Zheng, J. Zhao. "A novel RST-invariant digital image watermarking scheme", Third International Symposium on Multispectral Image Processing and Pattern Recognition, edited by Lu, Hanqing; Zhang, Tianxu, Proceedings of the SPIE, Vol. 5286, 2003, pp. 477-480.

[16] B. Kim, J. Choi, K. Park, F. Petitcolas, K. Hyoung Joong, "Image normalization using Invariant Centroid for RST Invariant digital image watermarking", First International workshop on digital watermarking, Seoul, Vol. 2613, 2002, pp. 202-211.

[17] J. Xuan, H. Zhang and L. Wang. "Rotation, scaling and translation invariant image watermarking based on Radon transform", Visual Communications and Image Processing, edited by Li, Shipeng; Pereira, Fernando; Shum, Heung-Yeung; Tescher, Andrew G., Proceedings of the SPIE, Vol. 5960, 2005, pp. 14991505.

[18] P. H. Wong, O. C. Au and Y.M. Yeung, "A Novel Blind Multiple Watermarking Technique for Images," EEE Transactions on Circuits and Systems for Video Technology, Vol. 13 No. 8, August 2003, pp. 813-830.

\section{BIOGRAPHY}

Kamel Mohamed Faraoun obtained a master's degree in Computer Science from the Computer Science department of Djilali Liabbes University Sidi-Bel-abbes - Algeria in 2002. His current research areas include computer safety systems, genetic algorithms, fractal images compression, evolutionary programming, grammatical inferences and physical materials structures modeling. He is currently a lecturer at the Computer Sciences Institute of Djilali Liabess University where he teaches operational researches and human-machine interaction. He is doing his Ph.D in the field of computer security using artificial intelligence systems. He has published several papers in international journals such as Genetic Fractal Images Compression (Multidimensional Systems and Signal Processing, Springer Science 2005). 
Rabhi Abbes obtained a master's degree in mathematics from the Mathematic Studies department of Djilali Liabbes University Sidi-Bel-abbes - Algeria in 2001. His current research areas include nonparametric estimation, statistical modelling and topological structures. He is currently a lecturer at the Mathematical Sciences Institute of Djilali Liabess University, he teaches probability theory, topological spaces and inductive logic, and is doing his $\mathrm{Ph} . \mathrm{D}$ in the field of nonparametric functional estimate infinite dimension. He has published several papers in international journals which include Indian Journal of Statistics Special Issue on Quantile Regression and Related Methods, 2005. 